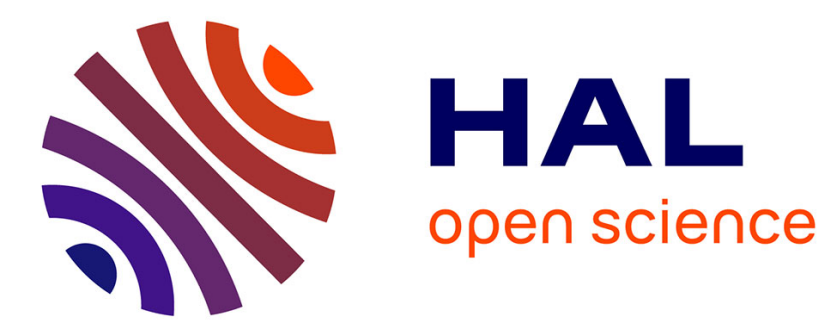

\title{
Effects of asynchronism on evolutionary games
}

Carlos Grilo, Luís Correia

\section{To cite this version:}

Carlos Grilo, Luís Correia. Effects of asynchronism on evolutionary games. Journal of Theoretical Biology, 2010, 269 (1), pp.109. 10.1016/j.jtbi.2010.10.022 . hal-00653673

\section{HAL Id: hal-00653673 https://hal.science/hal-00653673}

Submitted on 20 Dec 2011

HAL is a multi-disciplinary open access archive for the deposit and dissemination of scientific research documents, whether they are published or not. The documents may come from teaching and research institutions in France or abroad, or from public or private research centers.
L'archive ouverte pluridisciplinaire HAL, est destinée au dépôt et à la diffusion de documents scientifiques de niveau recherche, publiés ou non, émanant des établissements d'enseignement et de recherche français ou étrangers, des laboratoires publics ou privés. 


\title{
Effects of Asynchronism on Evolutionary Games
}

\author{
Carlos Grilo,a,b, Luís Correia ${ }^{\mathrm{b}}$ \\ ${ }^{a}$ Departamento de Engenharia Informática, Escola Superior de Tecnologia e Gestão, \\ Instituto Politécnico de Leiria, Morro do Lena, 2411-901 Leiria, Portugal. \\ ${ }^{b}$ LabMag, Departamento de Informática, Faculdade de Ciências da Universidade de \\ Lisboa, Edifício C6, Campo Grande, 1749-016 Lisboa, Portugal.
}

\begin{abstract}
We analyze the influence of the update dynamics on symmetric 2-player evolutionary games, which are among the most used tools to study the emergence of cooperation in populations of interacting agents. A synchronous dynamics means that, at each time step, all the agents of the population update their strategies simultaneously. An extreme case of asynchronism is sequential dynamics, in which only one agent is updated each time. We first show that these two opposite update dynamics can lead to very different outcomes and that sequential dynamics is detrimental to the emergence of cooperation only when the probability of imitating the most successful neighbors is high. In this sense, we can say that, when the update dynamics has some influence, in general asynchronism is beneficial to the emergence of cooperation. We then explore the consequences of using intermediate levels of asynchronism, where only a fraction of the agents update their behavior each time. In general, the level of cooperation changes smoothly and monotoni-
\end{abstract}

\footnotetext{
*Corresponding author

Email addresses: carlos.grilo@ipleiria.pt (Carlos Grilo), Luis.Correia@di.fc.ul.pt (Luís Correia)
} 
cally as we gradually go from synchronous to sequential dynamics. However, there are some exceptions that should be taken into account. In addition, the results show that the possibility of agents taking irrational decisions has a key role in the sensitivity of these models to changes in the update dynamics. Explanations for the observed behaviors are advanced.

Key words: Evolutionary game theory, Evolutionary games, Structured populations, Asynchronism, Artificial life

\section{Introduction}

The identification of mechanisms that promote emergence and maintenance of cooperative behaviors is of major relevance in a variety of scientific disciplines such as biology, sociology, economics and computer science (Nowak, 2006). Evolutionary game theory (Weibull, 1997) has been one of the main frameworks used to study this issue. In an evolutionary game the agents inside a population interact during several time steps through a given game which is used as a metaphor for the type of interaction that is being studied. The underlying structure that defines who interacts with whom is called the network of contacts or simply network. After each interaction session, some or all of the agents, depending on the update dynamics used, may update their strategies. If a synchronous dynamics is used, all the agents in the population update their strategies simultaneously at each time step. This models a strong correlation of the moments at which agents are updated. Such is not the case with asynchronous dynamics where only some agents, possibly just one, update their strategies at each time step. The strategy update process is modeled using a transition rule, which takes into 
account the payoffs collected by the agents during the interaction stage and emulates the fact that they tend to adapt their behavior to the context in which they live by imitating the most successful agents they know. It can also be interpreted as the selection step of an evolutionary process in which the least successful strategies tend to be replaced by the most successful ones. Note that, while the update dynamics defines the temporal relation between agents' update moments, thus modeling dynamics at a global level, the transition rule models local or individual dynamics. In this paper we study the effects of using different update dynamics on evolutionary games.

Perfect synchronism is an abstraction that presupposes the existence of a global clock by which the elements time their actions. In nature, this role can be played, for example, by year seasons or by the sun. In a variety of scientific disciplines, including the ones mentioned above, this has been the most common way of modeling the update dynamics of real systems. This practice has been widely questioned, the argument being that perfect synchronism is absent from the real world, even in systems where synchronization processes exist. For example, it is well known that populations of Pteroptyx malaccae fire-flies are able to synchronize so that they flash approximately at the same time (Winfree, 2001). However, actions being strongly correlated doesn't means that they are perfectly simultaneous. Furthermore, it has been shown that the dynamics and the patterns generated, for example, in cellular automata, of which evolutionary games are a special case, can be significantly affected if an asynchronous updating is used (Ingerson and Buvel, 1984; Bersini and Detours, 1994; Lumer and Nicolis, 1994; Fatès and Morvan, 2005). The most common alternative to synchronous updating is 
sequential updating, which is a special case of asynchronism: at each time step, exactly one element is updated. It has been shown that the order in which the elements are updated may also influence the dynamics and the final outcome of the system (Schönfisch and de Roos, 1999). Synchronous and sequential updating are seemingly opposite ways of modeling the update dynamics of a dynamical system. However, real systems, and biological ones in particular, seem to lie somewhere between these two extremes. Moreover, different systems may have distinct synchrony rates. Thus, in this paper we also investigate the consequences on evolutionary games of using an update method that allows the exploration of intermediate levels of asynchronism.

In Section 2, we review previous work on evolutionary games where this subject was studied. In sections 3 and 4, we present results using synchronous and sequential dynamics in order to show how the influence of the update dynamics varies when different conditions are used, namely different networks and transition rules which allow varying degrees of noise. We conclude that asynchronism is detrimental to cooperation for small noise values only. In Section 5 we explore intermediate levels of asynchronism in order to verify how much we can rely on the results obtained with synchronous and sequential dynamics and we discuss explanations for the way the update dynamics influences the emergence of cooperation. Finally, in Section 6 some conclusions are drawn and future work is advanced. 


\section{Previous Work}

Symmetric 2-player games are among the most studied games in evolutionary game theory. These games can be described by the payoff matrix

$$
\begin{gathered}
C \\
C\left(\begin{array}{ll}
R & S \\
T & P
\end{array}\right)
\end{gathered}
$$

where $\mathrm{C}$ (cooperate) and D (defect) are the possible actions for each player. Each element of the matrix represents the payoff received by the row-player when it plays the game against the column-player. Let us consider $R=1$ and $P=0$, and restrict $\mathrm{S}$ and $\mathrm{T}$ to the intervals $-1<S<1,0<T<2$ (Hauert, 2002; Roca et al., 2009a). The $S>0, T<1$ region corresponds to the Harmony game where the rational action for both players is to play $\mathrm{C}$ in a one shot game. The famous Prisoner's Dilemma game (Axelrod, 1984) corresponds to the region $S<0, T>1$. In this game there is a strong temptation to play D, which is the rational choice. However, if both players play $\mathrm{D}$, they receive a smaller payoff than if they both play $\mathrm{C}$, hence the dilemma. In the Snowdrift game, $S>0, T>1$, the best action depends on the opponent's decision: it is better to play $\mathrm{C}$ if the other player plays $\mathrm{D}$ and vice-versa. Finally, the region $S<0, T<1$ corresponds to the Stag-Hunt game. In this game there is a dilemma between playing the potentially more profitable but risky action $\mathrm{C}$ and the less profitable but less risky action $\mathrm{D}$.

In the classical approach of evolutionary game theory, agents are placed in a well-mixed infinite population where each agent may interact with any other and the proportion of cooperator and defector agents in the population evolves according to the replicator dynamics (Hofbauer and Sigmund, 
1998). In this setting, the asymptotic proportion of cooperator agents in the population, $\rho^{*}$, is 0 for the Prisoner's Dilemma game and 1 for the Harmony game. For the Snowdrift game we have $\rho^{*}=\rho_{e}$, where $\rho_{e}=S /(S+T-1)$. For the Stag-Hunt game, $\rho^{*}=0$ if $\rho^{0}<\rho_{e}$ and $\rho^{*}=1$ if $\rho^{0}>\rho_{e}$, where $\rho^{0}$ is the initial proportion of cooperators.

The work on evolutionary game theory has progressed over the years towards more realistic assumptions in the models used. One of the most important steps in this direction was taken by Nowak and May (1992), who showed that cooperation can be maintained when the Prisoner's Dilemma, with $R=1, T=b>1, S=P=0$, is played on a two-dimensional grid in which memoryless agents can only interact with their immediate neighbors. Regular grids reflect the fact that interactions in nature are conditioned by spatial distance. Spatial structure allows the formation of cooperator clusters. This way, cooperators interact mainly with each other while diminishing the contact and predation by defectors. However, regular grids do not account for the fact that, specially among humans, long range connections exist, which strongly reduce the distance between individuals otherwise far apart from each other in the grid. Small-world networks (Watts and Strogatz, 1998) model this property and the fact that real social networks have a strong local structure in the sense that two individuals with a common acquaintance probably also know each other. In real systems, it is also common that the number of connections varies greatly among the individuals of a population. The scale-free networks model (Barabási and Albert, 1999) was proposed to account for this property. While small-world networks are also known to support cooperative behaviors (Abramson and Kuperman, 2001; 
Tomassini et al., 2006; Vukov et al., 2008), it has been extensively reported that scale-free networks strongly enhance the survival of cooperation (Santos and Pacheco, 2005; Santos et al., 2005, 2006; Gómez-Gardeñes et al., 2007, 2008). Different aspects related to social structure have been studied such as, for example, clustering (Assenza et al., 2008), robustness to suppression of nodes (Perc, 2009), the existence of distinct interaction and updating neighborhoods (Ohtsuki et al., 2007), as well as the interplay between social structure and other aspects such as diversity of reproduction rates (Szolnoki et al., 2008c) and payoff normalization (Tomassini et al., 2007; Szolnoki et al., 2008b). Besides features related to social structure, as spatial distance and heterogeneity in the number of connections, it is commonly accepted that relatedness of individuals, reputation and repeated interactions, allied to memory and recognition capacities, are also mechanisms that promote cooperative behaviors (Axelrod, 1984).

The discussion about using synchronous or asynchronous dynamics on evolutionary games started with a paper by Huberman and Glance (1993), who contested the results achieved by Nowak and May (1992) due to the synchronous dynamics used. Huberman and Glance also presented results, apparently for a single payoff matrix, where cooperation was no longer sustainable when an asynchronous dynamics were used. Since then, this paper has become one of the most cited examples concerning the study of the consequences of asynchronous dynamics. These two studies used the besttakes-over transition rule, which dictates that each agent $x$ always imitates its most successful neighbor $y$, provided $y$ 's payoff is larger than $x$ 's payoff. After this, Nowak et al. (1994) tested their model under several conditions, 
including synchronous and sequential (asynchronous) dynamics, and showed that cooperation can be maintained for many different conditions, including asynchronism. They used a mathematical equivalent of what we call the Generalized Proportional (GP) transition rule: let $G_{x}$ be the aggregated payoff earned by agent $x$ in the present time-step; let $N_{x}^{*}=N_{x} \cup x$, where $N_{x}$ is the set of $x$ 's neighbors, with $k_{x}=\left|N_{x}\right|$; according to this rule, the probability that an agent $x$, with strategy $s_{x}$, imitates agent $y$, with strategy $s_{y}$, is given by

$$
p\left(s_{x} \rightarrow s_{y}\right)=\frac{\left(G_{y}-\Psi\right)^{\frac{1}{K}}}{\sum_{i}\left(G_{i}-\Psi\right)^{\frac{1}{K}}}, \quad y, i \in N_{x}^{*},
$$

where $K>0$ represents the noise present in the strategy update process. We can also interpret $1 / K$ as the selection intensity of the update process. $K \rightarrow 0$ corresponds to the best-takes-over rule described above. As $K$ grows, the probability that agents adopt strategies other than the one used by their most successful neighbor increases, as well as does the probability that they imitate agents less successful than themselves. For $K=1$ we have a linear proportional rule, also known as Moran rule. Finally, for $K \rightarrow+\infty$, payoffs play no role in the update process. The constant $\Psi$ is subtracted from the aggregated payoff because payoffs in the Stag-Hunt and the Prisoner's Dilemma games can be negative. If $G_{x}$ is set to the accumulated payoffs obtained by agent $x$ in the games played in the present time step, $\Psi=\max _{i \in N_{x}^{*}}\left(k_{i}\right) \min (0, S)$. If the average of the payoffs obtained in the present time step is considered instead, then $\Psi=\min (0, S)$.

Nowak et al. (1994) reported that, when $K \rightarrow 0$, sequential dynamics supports less cooperators than synchronous dynamics. On the other hand, when $K=1$, "cooperators seem to do better in the continuous [sequential] 
time simulations than in the discrete [synchronous] time simulations" (page 37). Recently, Newth and Cornforth (2009) studied the same model for the best-takes-over rule $(K \rightarrow 0)$ and confirmed the results reported by Nowak et al. (1994). They explored different types of asynchronous dynamics and found that they lead to different dynamical features as, for example, random walking gliders, not observed in the synchronous case.

The effect of the update dynamics on the Snowdrift game was also studied when it is played on regular (Hauert and Doebeli, 2004, see also the supplementary material) and small-world networks (Tomassini et al., 2006), with payoffs equivalent to $R=1, S=1-r, T=1+r$ and $P=0$, where $0 \leq r \leq 1$ represents the cost-to-benefit ratio of mutual cooperation. The results for the best-takes-over and Moran rules are qualitatively similar to the ones described above for the Prisoner's Dilemma game. However, there is a less intense influence of the update dynamics in the Snowdrift game. Both these studies used also, as transition rule, the replicator rule, which is the replicator dynamics version for finite populations and discrete time (Helbing, 1992; Schlag, 1998) described below. They found that, with this rule, the level of cooperation is not affected by the update dynamics that is used and the same was reported for both the Prisoner's Dilemma and Snowdrift games played on scale-free networks (Santos et al., 2005).

In the replicator rule, the probability that an agent $x$ imitates a randomly chosen neighbor $y$ is given by $p\left(s_{x} \rightarrow s_{y}\right)=\left(G_{y}-G_{x}\right) / \Phi$, provided that $G_{y}-G_{x}>0$. The constant $\Phi$ assures $p\left(s_{x} \rightarrow s_{y}\right) \in[0,1]$. If accumulated payoffs are used, then $\Phi=\max \left(k_{x}, k_{y}\right)[\max (1, T-\min (0, S))]$. If average payoffs are considered, then $\Phi=\max [1, T-\min (0, S)]$. We note that in 
this rule agents do not imitate neighbors with lower payoffs. Rules with this property are called payoff monotone (Szabó and Fáth, 2007).

Finally, in a recent paper, Roca et al. (2009a) studied the influence of the update dynamics in the $S T$-plane, covering the four games described in the beginning of this section, using regular and small-world networks and different transitions rules, among which, the best-takes-over, Moran and replicator transition rules. The results are consistent with previous works but the authors conclude that, in this more general setting, the influence of the update dynamics is the exception rather than the rule, and that this influence is very dependent on the transition rule used. More specifically, they found that, for the replicator rule, the results obtained with synchronous and sequential dynamics are very similar and that the differences observed for the best-takes-over and Moran rules are limited to a small part of the ST-plane. In another paper, (Roca et al., 2009b), the same authors reported a large difference between the proportion of cooperators obtained with synchronous and sequential dynamics in the limit of small noise when scale-free networks are used. In this case, sequential dynamics favors cooperation, which is contrary to previous results obtained on regular grids and small-world networks with the best-takes-over rule.

We find thus that different results were obtained with distinct transition rules and networks. This shows that there is not yet a full understanding of the role played by the update dynamics on evolutionary games. For example, given that the transition rules commonly used correspond to very different levels of noise, we do not know if there is a general tendency for the way in which the update dynamics influences the emergence of cooperation. Hence, 
we need to explore intermediate levels of noise in order to verify if a tendency exists. This will be the subject of Section 4. As a first approach, in the next section we present results using the usual transition rules.

\section{Synchronous and Sequential Dynamics in the ST Plane}

We use two types of networks: small-world networks (Watts and Strogatz, 1998) and scale-free networks (Barabási and Albert, 1999). In order to build small-world networks, first a toroidal regular 2D grid is built so that each node is linked to its 8 surrounding neighbors by undirected links; then, with probability $\phi$, each link is replaced by another one linking two randomly selected nodes. Self links, repeated links and disconnected graphs are not allowed. These networks have the property that, even for very small $\phi>0$ values, the average path length is much smaller than in a regular network, maintaining a high clustering coefficient, which measures the average probability that the neighbors of a node are also connected. Both these properties are very commonly observed in real social systems. As $\phi \rightarrow 1$, we get random networks with both small average path lengths and clustering coefficients. The values used in the experiments were $\phi=\{0,0.01,0.05,0.1,1\}$. Scale-free networks are built in the following way: the network is initialized with $m$ fully connected nodes. Then, new nodes are added, one at a time, until the network has the desired size. Each added node is linked to $m$ already existing nodes so that the probability of creating a link to some existing node $i$ is equal to $k_{i} / \sum_{j} k_{j}$, where $k_{i}$ is the degree of $i$, which is defined as the number of nodes to which it is connected. This method of link creation leads to a power law degree distribution $P(k) \sim k^{-\gamma}$ that is very common in 
real social networks. All the networks for which results are presented have average degree $\bar{k}=8$ (equivalent to $m=4$ in scale-free networks).

In the synchronous model, at each time step all the agents play a one round game with all their neighbors and they collect the payoffs resulting from these games. After this, they all simultaneously update their strategies using the transition rule. In the sequential system, at each time step, one agent $x$ is randomly selected; $x$ and its neighbors play the game with their neighbors and, after this, $x$ updates its strategy. We used both accumulated and average payoffs. For small-world networks, the results obtained with the two approaches are similar since all the agents have approximately the same $k$ ( $k$ is the same for all agents when $\phi=0$ ). Major differences appear for scale-free networks due to a large heterogeneity in $k$ values. Average payoffs are intended to model the fact that agents have limitations in the number of interactions they can sustain simultaneously and also that relationships are costly (Tomassini et al., 2007; Szolnoki et al., 2008b).

The charts presented were obtained with populations of $n=10^{4}$ agents, randomly initialized with $\rho^{0}=0.5$. We let the system run during $10^{4}$ time steps for the synchronous model and $10^{4} \times n$ time steps for the sequential model, which is enough for the system to converge to homogeneous populations of cooperators or defectors, or to stabilize around a $\rho$ value. The steady state $\rho$ value is computed as the average proportion of cooperators in the last $10^{3}$ time steps for the synchronous model and in the last $10^{3} \times n$ time steps for the sequential model. Each point in the charts presented is an average of 50 independent simulations. For each simulation a new network is generated, which is kept static during the evolutionary process. 
Fig. 1 shows results obtained with regular grids and the replicator, besttakes-over and Moran transition rules, as in (Roca et al., 2009a). The results confirm the ones of previous works: there are no relevant differences between synchronous and sequential dynamics for the replicator rule; sequential dynamics supports less cooperation for the best-takes-over rule, with the exception of the Stag-Hunt game; sequential dynamics supports more cooperation for the Moran rule. With the best-takes-over rule, the main differences appear in the Snowdrift game, while there are no large differences in the Stag-Hunt and Prisoner's Dilemma games. The main differences appear for the Moran rule, especially in the Snowdrift and Stag-Hunt games. The influence in the Prisoner's Dilemma game is limited to a small region. However, in this region, synchronous dynamics leads to uniform populations of defectors, while sequential dynamics leads to populations strongly dominated by cooperators or even states where $\rho=1$. This is also the case of the Stag-Hunt and Snowdrift games for a noticeable portion of the space. For the Stag-Hunt this difference is a natural one, due to its bi-stable nature. However, that is not the case with the Prisoner's Dilemma and Snowdrift games, which makes this influence more relevant.

The main differences between the results obtained with regular and smallworld networks exist for the best-takes-over rule: in the Snowdrift and Prisoner's Dilemma games, sequential dynamics becomes progressively beneficial to cooperation as $\phi$ is increased. For $\phi=0.05$, sequential dynamics already supports more cooperation on average than synchronous dynamics when the best-takes-over rule is used (Fig. 2).

When scale-free networks are used, there are no relevant differences be- 

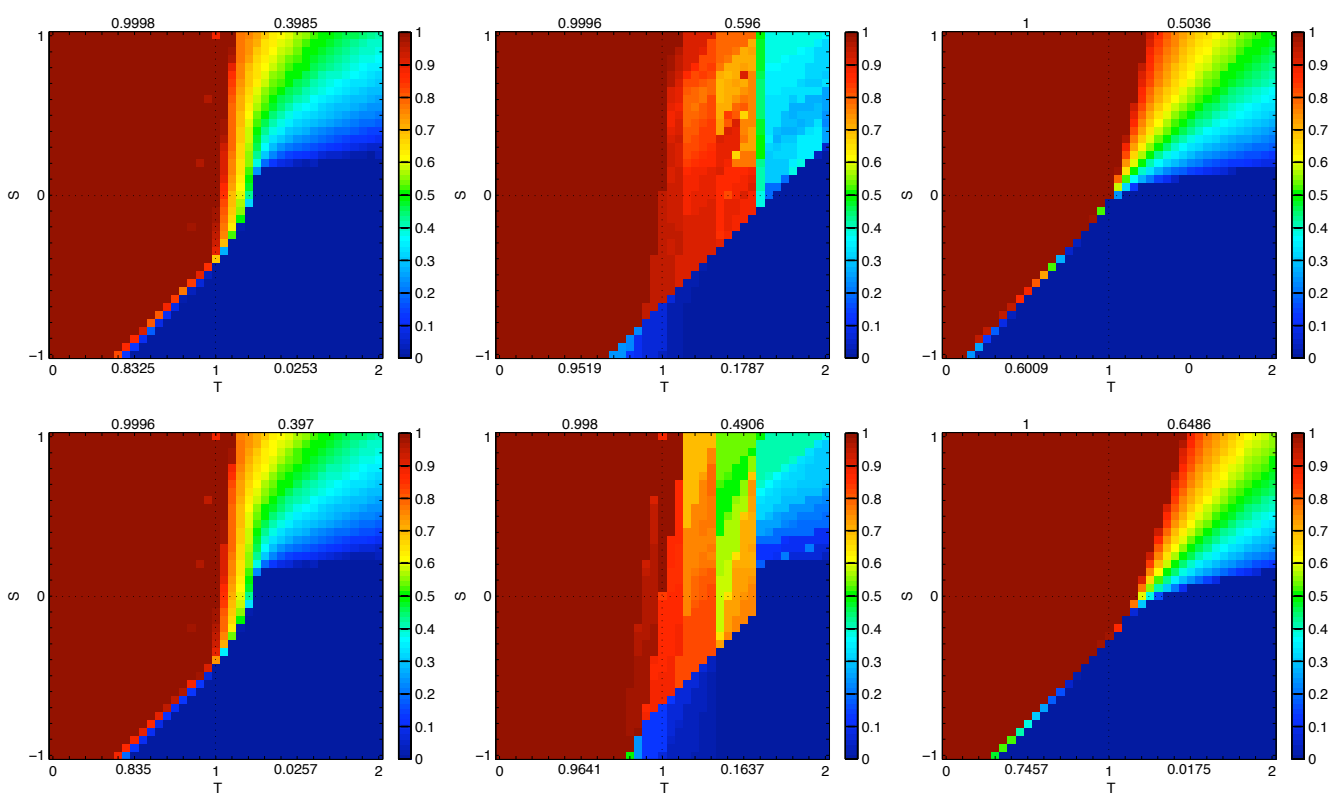

Figure 1: Proportion of cooperators $\rho$ in regular grids $(\phi=0)$, with synchronous dynamics (upper row) and sequential dynamics (lower row). The transition rules are the replicator rule (left column), the best-takes-over rule (middle column) and the Moran rule (right column). The numbers, respectively, above the Harmony and Snowdrift games, and below the Stag-Hunt and the Prisoner's Dilemma games, are the average values of the corresponding quadrant. The $S$ and $T$ parameters are varied in steps of 0.05 . For the replicator rule, there are no relevant differences between synchronous and sequential dynamics. Differences exist for the best-takes-over and Moran rules and there are many situations in which the update dynamics makes the difference between steady states where only defectors exist and states where cooperators and defectors coexist or even only cooperators survive.

tween sequential and synchronous dynamics when the replicator rule is used, as in regular and small-world networks. However, there are relevant differences for the best-takes-over and Moran rules. The differences are larger for accumulated payoffs (Fig. 3) than for average payoffs (Fig. 4). In the first 

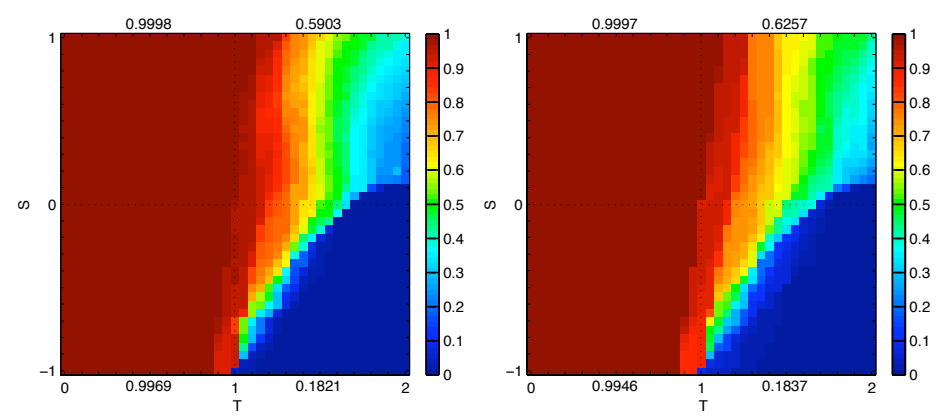

Figure 2: Proportion of cooperators $\rho$ on small-world networks $(\phi=0.05)$ with the besttakes-over rule. Left column: synchronous dynamics; Right column: sequential dynamics. In the Snowdrift and Prisoner's Dilemma games, sequential dynamics becomes progressively beneficial to cooperation as $\phi$ is increased. For $\phi=0.05$, sequential dynamics already supports more cooperation on average than synchronous dynamics when the besttakes-over rule is used. Concerning the Moran rule (not shown), there are no significant differences to regular grids.

case, and for the best-takes-over rule, cooperation completely dominates for the whole quadrant corresponding to the Snowdrift game when sequential dynamics is used. For the Stag-Hunt and Prisoner's Dilemma games, sequential dynamics leads to a significant increment of cooperation in large portions of the space. We note also that with these networks, when the update dynamics has some influence over $\rho$, sequential updating is always beneficial to cooperation when accumulated payoffs are used, with only a few exceptions to this behavior when average payoffs are used.

These results suggest that asynchronous updating is beneficial to the emergence of cooperation more often than it is detrimental. More specifically, they suggest that asynchronism is detrimental to cooperation only for networks with a high degree of regularity and for low or no noise. In the next 

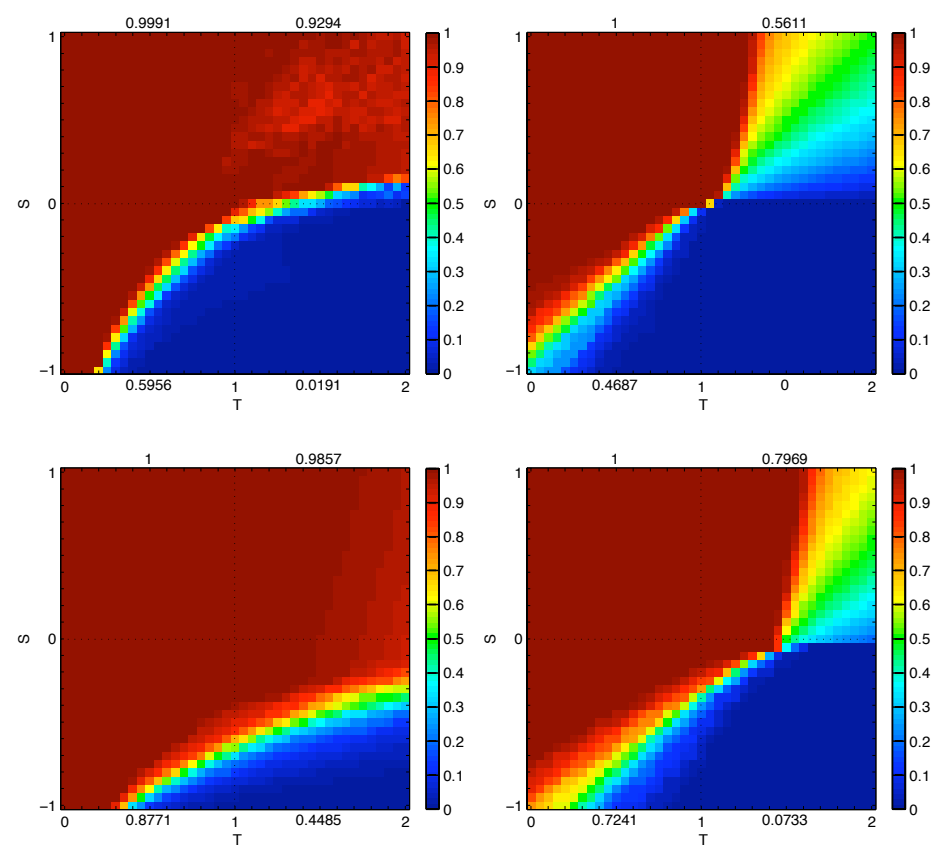

Figure 3: Proportion of cooperators $\rho$ in scale-free networks $(m=4)$, with synchronous dynamics (upper row) and sequential dynamics (lower row) using accumulated payoffs. Left charts: best-takes-over rule; Right charts: Moran rule. With both rules, there are relevant differences between synchronous and sequential dynamics. With these networks, sequential dynamics never supports less cooperators than the synchronous counterpart.

section we explore intermediate levels of noise in order to verify this.

\section{Intermediate Noise Levels}

We use the GP transition rule (Equation 2), and the Fermi transition rule (Szabó and Fáth, 2007) in order to model intermediate noise levels in the strategy update process. The neighborhood monitoring degree is different in the two rules. While the GP rule takes into account the payoffs of all the neighbors, the Fermi rule only evaluates the payoff of one neigh- 

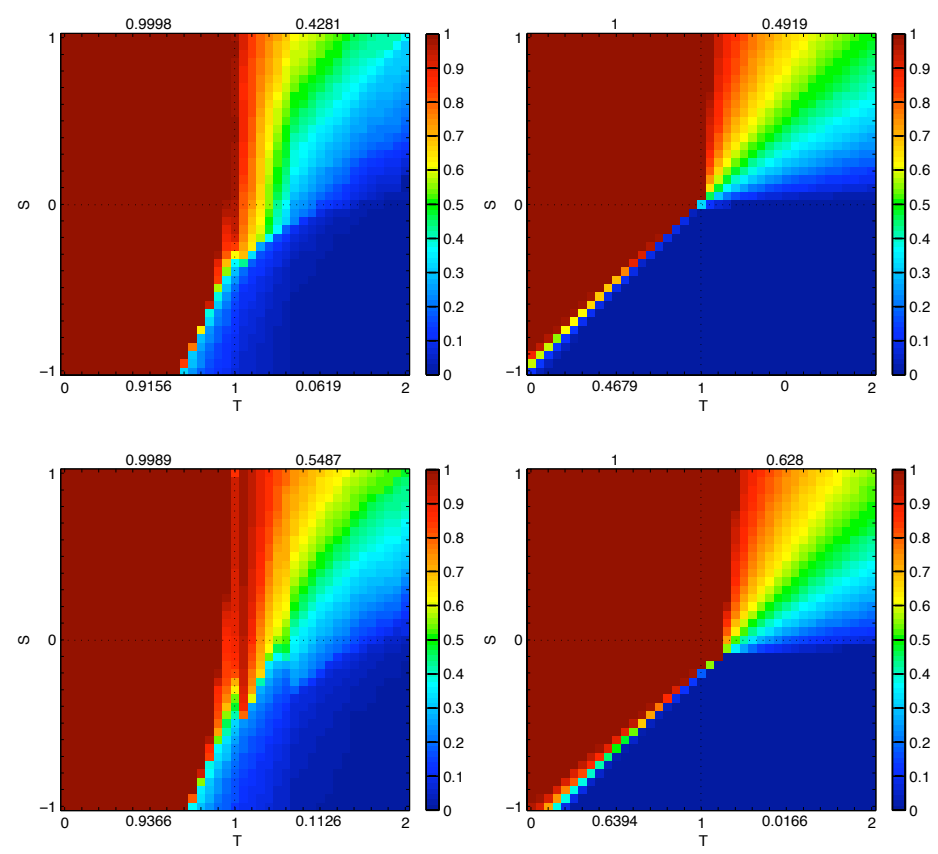

Figure 4: As in Fig. 3 but with average payoffs. The differences are not as relevant as with accumulated payoffs but we note that, in many situations, the update dynamics makes the difference between the coexistence of the two strategies and uniform steady states. We note also that, excepting a few cases for the best-takes-over rule, sequential dynamics is not detrimental to cooperation.

bor. According to the Fermi rule, the probability that an agent $x$ imitates a randomly chosen neighbor $y$ is equal to

$$
p\left(s_{x} \rightarrow s_{y}\right)=\frac{1}{1+\exp \left[-\left(G_{y}-G_{x}\right) / K\right]},
$$

where $K>0$ represents the noise present in the strategy update process. As in the GP rule, as $K$ grows, the probability that agents imitate less successful neighbors increases. However, differently from the GP rule, $K \rightarrow 0$ does not imply the imitation of the most successful neighbor since $y$ is ran- 
domly chosen. That is, $K \rightarrow 0$ in the Fermi rule models a smaller selection intensity than the GP rule with $K \rightarrow 0$. For the GP rule, we use $K \in\{0,1 / 100,1 / 10,1 / 8,1 / 6,1 / 4,1 / 2,1\}$. We use $K \in[0,0.1]$ in steps of 0.01 for the Fermi rule with the Prisoner's Dilemma and $K \in[0,1]$ in steps of 0.1 with the Snowdrift game. We use a larger interval with the Snowdrift game since it is less sensitive to noise than the Prisoner's Dilemma game.

We used the typical one parameter versions of the Prisoner's Dilemma and Snowdrift games: $R=1, T=b \in[1,2], S=P=0$ for the Prisoner's Dilemma game, and $R=1, S=1-r, T=1+r, P=0$, with $r \in[0,1]$, for the Snowdrift game. These versions were used since they allow for comparisons with previous works and because the conclusions that can be driven for these subsets, with the transition rules used in the previous section, are representative of the conclusions that can be driven for the respective game in the $S T$-plane. Here, we concentrate on these two games because the results obtained with the Stag-Hunt game show that it answers very coherently to the modification from synchronous to sequential dynamics: when this change has some significant influence, asynchronous dynamics benefits cooperation.

Let us consider the difference $\Delta \rho=\rho_{\text {seq }}-\rho_{\text {sync }}$, where $\rho_{\text {seq }}$ and $\rho_{\text {sync }}$ are, respectively, $\rho$ values obtained with sequential and synchronous dynamics for the same set of parameters (game, network and noise parameters). Positive or negative $\Delta \rho$ values mean that sequential dynamics supports, respectively, more or less cooperators than synchronous dynamics.

Fig. 5 shows results for the Prisoner's Dilemma and Snowdrift games played with the GP and the Fermi rules on regular networks, which is the case in which $\Delta \rho<0$ occurs more often. For the GP rule, these situations 

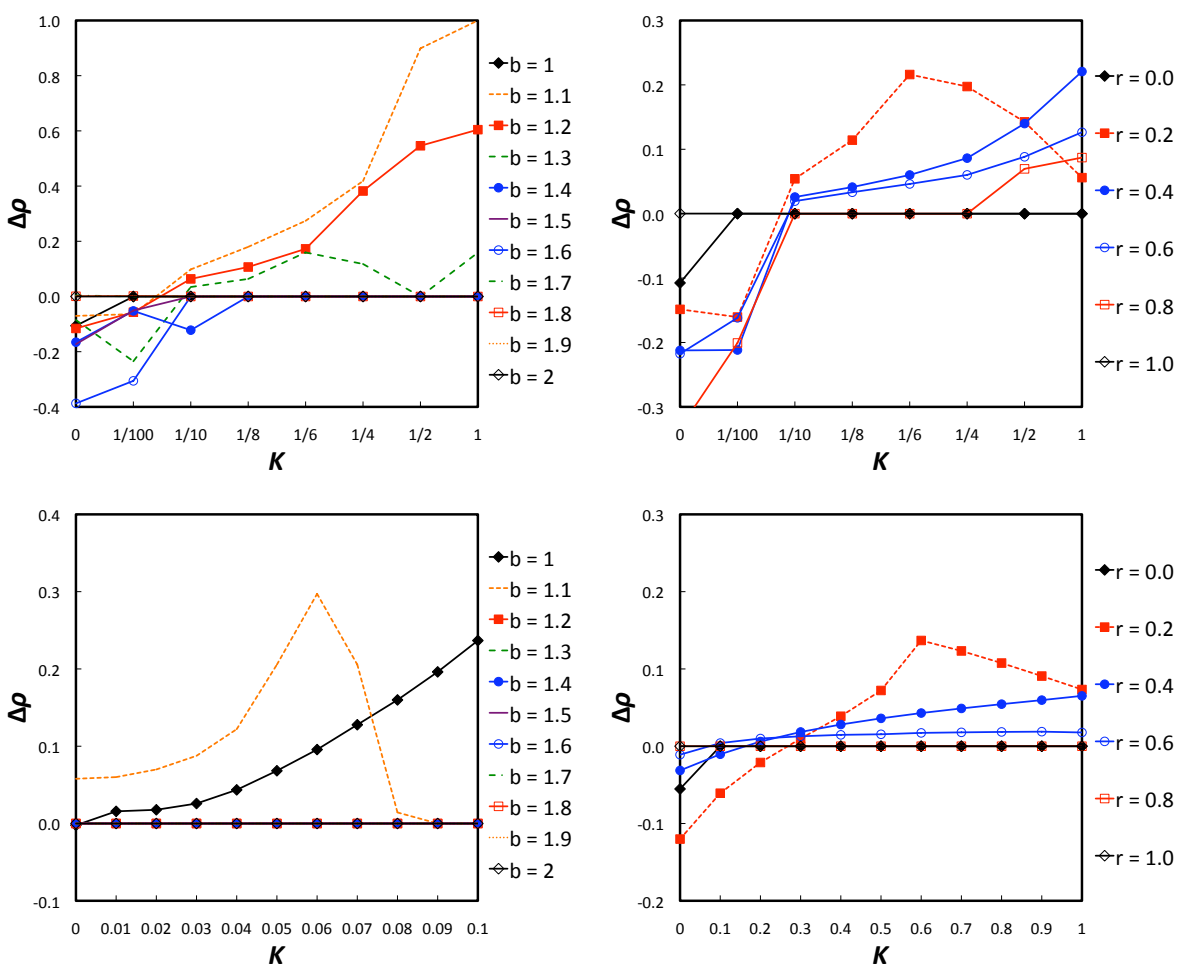

Figure 5: $\Delta \rho$ values obtained with the GP rule (upper row) and the Fermi rule (lower row), when the games are played on regular grids. Left charts: Prisoner's Dilemma; Right charts: Snowdrift. For the Snowdrift charts, only $\Delta \rho$ values for even $r$ values are shown in order to improve readability. Sequential dynamics is detrimental to cooperation $(\Delta \rho<0)$ mainly when regular networks are used and only for small noise values. The Fermi rule is less sensitive to the update dynamics than the GP rule and, for the Prisoner's Dilemma game, it also supports less cooperation: the most part of the $\Delta \rho$ values is equal to 0 because $\rho=0$ for both synchronous and sequential dynamics. Notice the different scales.

occur for $K \leq 1 / 10$ only and they diminish in quantity and intensity for both games as the rewiring probability, $\phi$, is increased. On scale-free networks, $\Delta \rho<0$ occurs only when average payoffs are used, and just for the extreme cases of $b=1$ (Prisoner's Dilemma) and $r=0$ (Snowdrift) with $K \rightarrow 0$. 
The results for the Fermi rule are in line with the ones obtained with the GP rule: $\Delta \rho<0$ occurs only for smaller $K$ values and mainly for the Snowdrift game. However, the Fermi rule is less sensitive to the update dynamics. That is, $|\Delta \rho|$ values are, in general, smaller for the Fermi rule.

We can also observe in Fig. 5 that, for some $b$ and $r$ values, $\Delta \rho$ diminishes as $K$ grows above some value. This happens for both rules and is related to the direct influence of $K$ over $\rho$. In these situations, as $K$ grows, the value of $\rho$ approaches, and sometimes reaches, 0 or 1 for sequential or synchronous dynamics (and, sometimes, for both). As a side effect, the influence of the update dynamics diminishes for larger $K$ values. However, we note that, even in these situations, $\Delta \rho$ never becomes negative.

The results reported in this section show that $\Delta \rho<0$ occurs for small noise values only and mainly for regular grids. This means that, in general, when the update dynamics has some influence, sequential dynamics supports more cooperators than synchronous dynamics. While the results shown are completely coherent with the ones reported in previous studies, the conclusion is different due to the exploration of different types of networks and intermediate values of noise present in the strategy update process, modeled with two different transition rules.

\section{Intermediate Levels of Asynchronism}

In order to model intermediate levels of asynchronism, we use a parameter $\alpha$, which represents the synchrony rate of the model (Fatès and Morvan, 2005). At each iteration, each agent is chosen with probability $\alpha$. The chosen agents and their neighbors play the game with their neighbors. Then, 
the chosen agents synchronously update their strategies using the transition rule. This method allows us to cover all the space between synchronous and sequential dynamics: $\alpha=1$ corresponds to synchronous dynamics; as $\alpha \rightarrow \frac{1}{n}$, where $n$ is the population size, the model approaches random sequential dynamics. Experiments where $\alpha \neq 1$ are setup so that the number of individual updates is approximately the same as in the $\alpha=1$ case. More specifically, each simulation is stopped after the first time step where the number of individual updates exceeds $n * 10^{4}$, while the measurement of $\rho$ starts in the first time step where the number of individual updates exceeds $n * 9 \times 10^{3}$.

\subsection{Monotonicity and Local Sensitivity of $\rho$ in relation to $\alpha$}

We first verified if $\rho$ varies monotonically as we change $\alpha$ in one direction (Grilo and Correia, 2009). When the Fermi rule is used, $\rho$ always changes monotonically with $\alpha$, no matter the noise level or the network used. When the GP rule is used instead, non-monotonicity arises for both games mainly when regular grids and small noise values are used (Fig. 6) and it diminishes, and eventually disappears, as both the noise level $K$ and the rewiring probability $\phi$ grow. These results taken together, namely, the fact that nonmonotonicity occurs mainly for regular networks and the fact that selection intensity is not as strong in the Fermi rule as in the GP rule when $K \rightarrow 0$, suggest that non-monotonicity of $\rho$ in relation to $\alpha$ is mainly associated with regular networks and high selection intensity. This means that, in general,

the results obtained with synchronous and sequential dynamics give us a fair idea of the maximum influence the update dynamics can have on $\rho$.

However, if we use intermediate asynchronicity levels, we notice a complex behavior, mainly when the difference between the $\rho$ value obtained with 

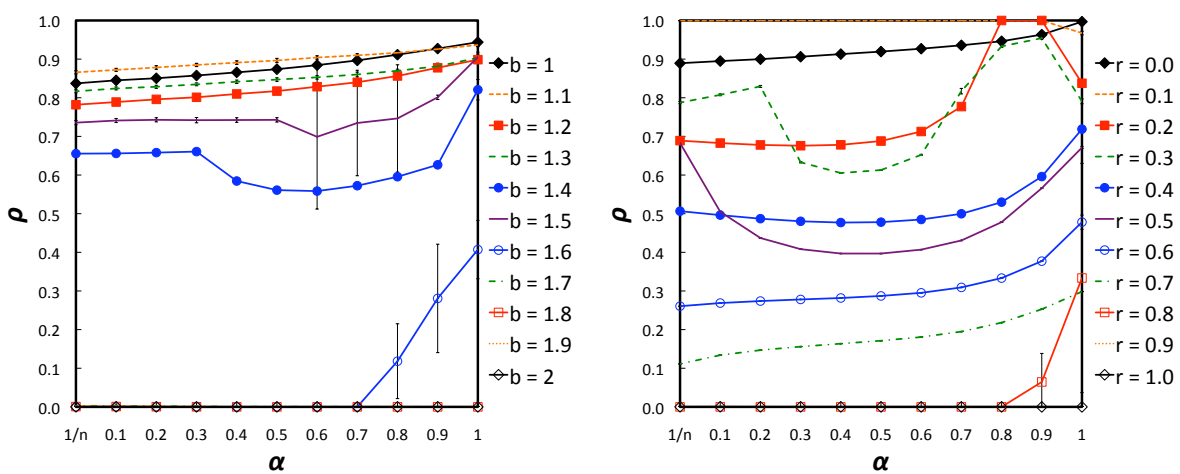

Figure 6: $\rho$ values obtained with the best-takes-over rule (GP rule with $K \rightarrow 0$ ) when the Prisoner's Dilemma (left) and Snowdrift (right) games are played on regular grids. The most intense situations of non-monotonicity occur for low noise values and regular grids.

sequential and synchronous dynamics is large: the variation of $\rho$ as $\alpha$ is changed from 1 to $1 / n$ is not always smooth. Indeed, there are situations where $\rho$ is approximately the same for a large portion of the $\alpha$ domain, changing abruptly at a certain point when $\alpha$ is changed by a small value (threshold point). The most impressive examples occur when both games are played on scale-free networks with the GP rule and accumulated payoffs. As Fig. 7 shows, there are many situations of strong sensitivity when $\alpha$ is diminished by a small value, specially when it is changed from 1 (synchronous dynamics) to 0.9 . These situations occur for $K \in[0,1 / 2]$. For $K=1$, the transitions become smoother. We verified also that, in these cases, the dynamics is different for $K \leq 1 / 100$ and for $K>1 / 100$. In the first case, the system converges to fixed states or to cyclic dynamics. Decreasing the synchrony rate $\alpha$ prevents the population from becoming trapped in low $\rho$ fixed states/cyclic dynamics. For larger $K$ values, we observed that the propor- 
tion of cooperators oscilates during the evolutionary process, with constant and fast transitions between states where cooperators strongly dominate and states where they are in clear minority. Eventually, the population converges to uniform states of cooperators (all-C) or defectors (all-D). Given this behavior, it is impressive that decreasing $\alpha$ by a small value frequently makes the difference between systematic convergence to all- $\mathrm{C}$ or systematic convergence to all-D. This may prefigure the occurrence of second-order phase transitions (Fatès, 2009).

\subsection{Payoff Monotonicity}

In this section we focus on the features of the transition rules that may influence the sensitivity of evolutionary games to the update dynamics. We start by recalling that these games are completely insensitive to the update dynamics when the replicator rule is used (see Section 3). This rule is payoff monotonous, i.e., it does not allow the imitation of less successful neighbors. It models also a lower selection intensity than both the GP and Fermi rules with $K \rightarrow 0$ : in this regime, these rules, being also payoff monotone, are nevertheless sensitive to the update dynamics. This suggests that insensitivity to the update dynamics is associated with both payoff monotonicity and low selection intensity.

In order to verify this, we modified the GP rule so that agent $y$, chosen with Equation 2, is imitated by focal agent $x$ only if it has a larger aggregated payoff than $x$. Fig. 8 exemplifies the differences between the original and the modified GP rule when $K=1$ for both games played on small-world networks with $\phi=0.1$. It shows that for such a low selection intensity, both games become less sensitive when the modified GP rule is used. This con- 

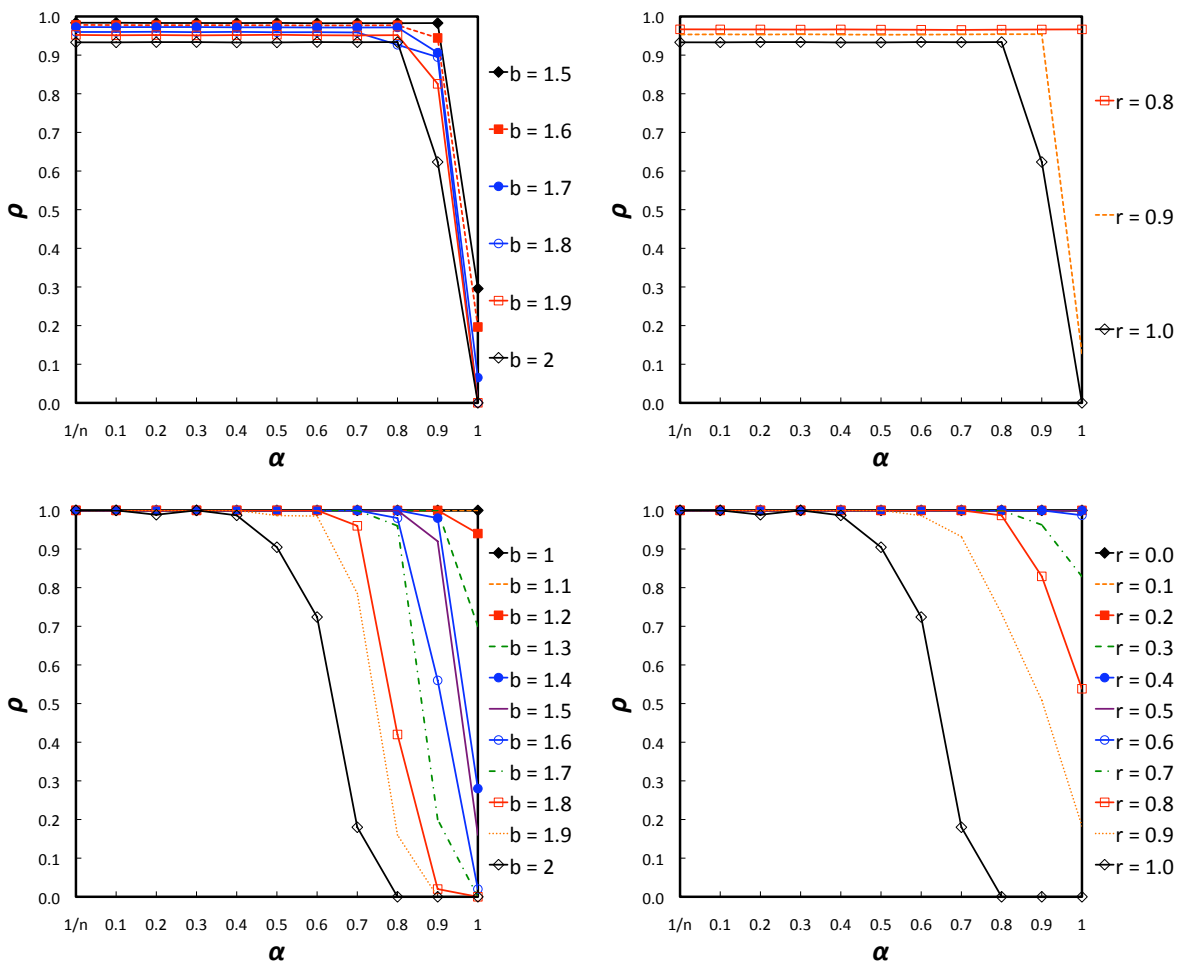

Figure 7: $\rho$ values obtained with the Prisoner's Dilemma (left column) and Snowdrift (right column) played on scale-free-networks with the GP rule and accumulated payoffs. Upper row: best-takes-over rule (GP rule with $K \rightarrow 0$ ); Lower row: GP rule with $K=1 / 2$. Only some lines are shown in the upper row charts in order to improve readability. Both games are strongly sensitive to small $\alpha$ changes, mainly for larger $b$ (Prisoner's Dilemma) and $r$ (Snowdrift) values and specially in the transition from $\alpha=1$ to $\alpha=0.9$.

firms that payoff monotonicity associated with low selection intensity is a key property concerning the insensitivity of these games to the synchrony rate $\alpha$. The fact that the Fermi rule is less sensitive than the GP rule (see Section 4), suggests that the neighborhood monitoring degree is also an important feature concerning sensitivity to the update dynamics. However, the results 

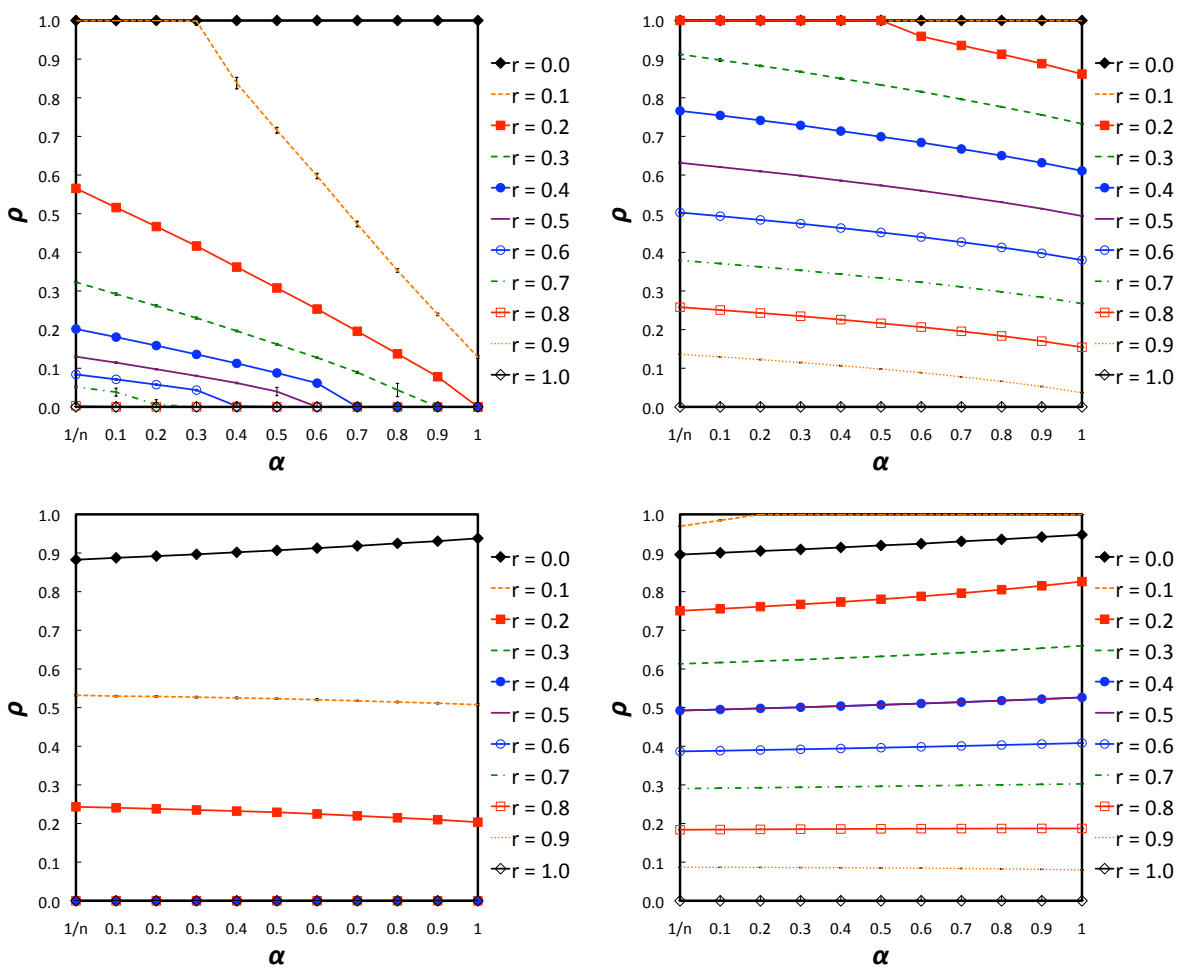

Figure 8: $\rho$ values obtained for the original and the modified GP rule with $K=1$ when the Prisoner's Dilemma (left column) and Snowdrift (right column) are played on small-world networks with $\phi=0.1$. Upper row: original GP rule; lower row: modified GP rule. Both games become less sensitive to the synchrony rate when payoff monotonicity is introduced.

presented in (Roca et al., 2009a) for the multiple replicator rule, which is a version of the replicator rule that models a complete neighborhood monitoring, show that it is also insensitive to the update dynamics. This means that, while payoff monotonicity associated with low selection intensity is sufficient to turn both games insensitive to the update dynamics, a complete neighborhood monitoring is not sufficient to turn them sensitive. 


\subsection{Strategy Exchanges and (A)synchronism}

In order to understand why smaller synchrony rates lead to more cooperation when agents are allowed to take irrational choices, we depart from an idea from Tomassini et al. (2006) stated for the Snowdrift game. They noted that, when the Moran rule is used (GP rule with $K=1$ ), more cooperators survive with sequential dynamics than with the synchronous counterpart. Their intuitive explanation is the following: With sequential dynamics, if a defector imitates a cooperator neighbor, the proportion of cooperators increases and, furthermore, the next time one of them is evaluated for an update, it will take advantage of the other's presence to increase its payoff. On the other hand, if a cooperator imitates a defector neighbor, the two agents will negatively influence each other's payoff. According to the authors, the same reasoning can not be applied to synchronous dynamics because cooperator and defector neighbors can exchange their strategies at the same time step, thus preventing the new cooperator to take advantage of its neighbor's presence.

In order to verify this idea, we first measured the average number of strategy exchanges, as a function of $\alpha$. Two neighbor agents $x$ and $y$ exchange their strategies at time step $t$ if conditions $s_{x}=S_{A} \wedge s_{y}=S_{B}$ and $s_{x}=S_{B} \wedge s_{y}=S_{A}$, with $S_{A} \neq S_{B}$ hold before and after the strategy update process, respectively. The average number of strategy exchanges is taken over periods of $\frac{1}{\alpha}$ time steps so that the number of individual updates considered is approximately the same as for the synchronous case $(\alpha=1)$. Thus, strategy exchanges are, measured for $\alpha \in\{1,0.5,0.2,0.1,1 / n\}$ only.

The observed result is a decrease in the number of strategy exchanges as 
$\alpha$ decreases (see Fig. 9 for the GP rule). An exception to this behavior occurs when the population converges to a state where only defectors survive or only a very small fraction of cooperators survive for some $\alpha$ value. This happens mainly in the Prisoner's Dilemma game for larger $K$ values. For example, in Fig. 9 (c) we can see that for $b=1.2$, the number of strategy exchanges is 0 for $\alpha=1$ due to the extinction of cooperators (see the upper left chart of Fig. 8). Apart from these situations, the number of strategy exchanges always increases with $\alpha$. Fig. 9 also shows that the number of strategy exchanges is larger for $K=1$ than for $K \rightarrow 0$, except for the situation already mentioned. This increase occurs for all networks in conjunction with average payoffs. When scale-free networks are used with accumulated payoffs, the number of strategy exchanges is always very low, independent of the noise values. In this case, payoffs can vary substantially from agent to agent, which makes strategy exchanges less probable. Thus, the results described in the remaining of this section and in the following one do not apply to this case.

The results for the Fermi rule are qualitatively similar to the ones obtained with the GP rule. However, the differences between the number of strategy exchanges obtained with different $K$ values are smaller, which is consistent with the smaller sensitivity of the Fermi rule. Concerning the replicator rule, the number of strategy exchanges is always very small for both games, never exceeding 16 exchanges per $\frac{1}{\alpha}$ period, no matter the $\alpha$ value used. These results combined with the beneficial effect of asynchronism, verified when noise is present in the GP and the Fermi rules, as well as the insensitivity of the replicator rule, suggest indeed a close relationship between the number of strategy exchanges and the way $\rho$ reacts to $\alpha$ changes. However, this does 

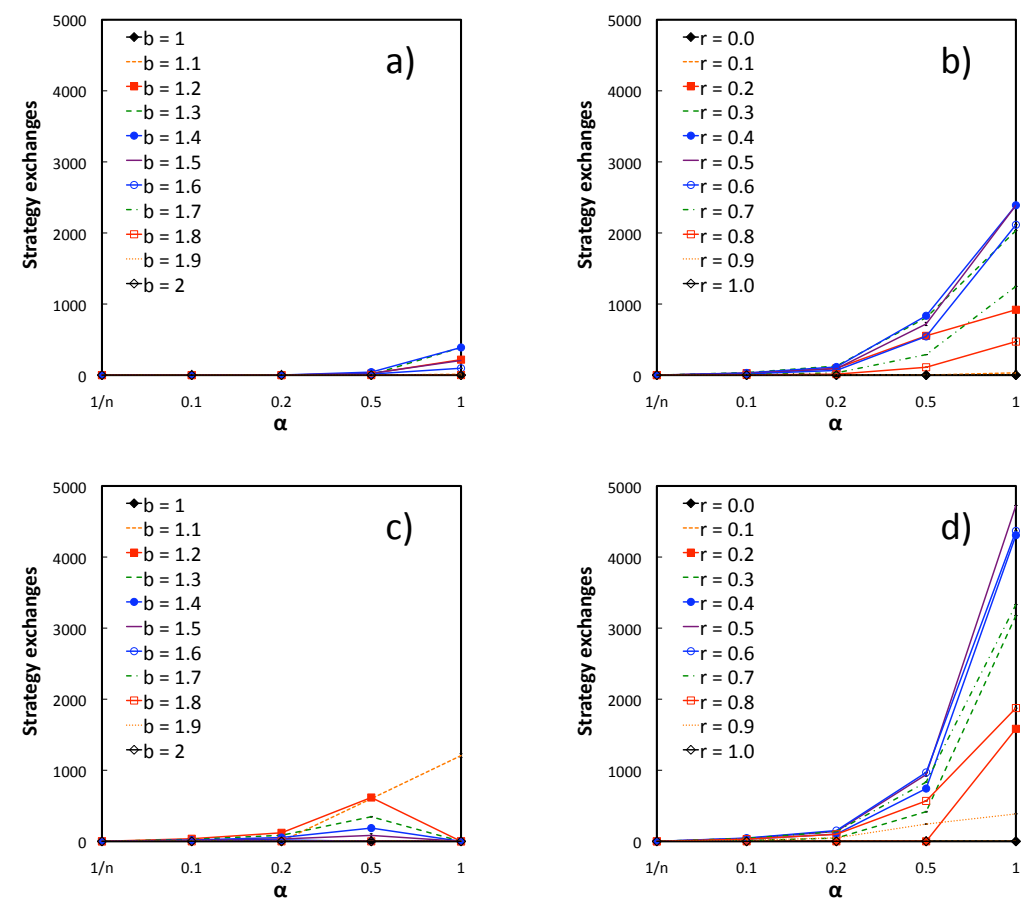

Figure 9: Number of strategy exchanges as a function of $\alpha$, when the Prisoner's Dilemma (left column) and Snowdrift (right column) are played on small-world networks $(\phi=0.1)$ using the GP rule. Upper row: $K \rightarrow 0$ (best-takes-over rule); Lower row: $K=1$ (Moran rule). Strategy exchanges diminish as $\alpha$ is decreased and are larger for $K=1$ than for $K \rightarrow 0$.

not allow us to establish a cause-effect relationship between the number of strategy exchanges and $\rho$ since they are both dependent variables.

\subsection{Effects of Strategy Exchanges}

In order to verify if there is a cause-effect relationship between the number of strategy exchanges and the value of $\rho$, we modified the model so that, at each time step, either a randomly chosen agent is updated with probability $1-p$ using the transition rule or two randomly chosen neighbor agents 
exchange their strategies with probability $p$. We note that no strategy exchanges can occur as a result of the application of the transition rule since only one agent is selected. That is, when $p=0$, this is a sequential model. Fig. 10 exemplifies the effect of strategy exchanges on the value of $\rho$ when both games are played on regular networks with the GP and the replicator rules. It shows that $\rho$ decreases as $p$ increases, except for the Snowdrift game played on small-world networks with the replicator rule.

The difference between situations where the games are sensitive to $p$ and situations where they are not can be understood if we look at the spatial patterns formed by the agents during the evolutionary process. Figs. 11 and 12 show examples, respectively for the Prisoner's Dilemma and Snowdrift games, of spatial patterns formed with the GP and replicator rules, for $p=0$ and $p \neq 0$. With the exception of the Snowdrift played under the replicator rule (Figs. $12 \mathrm{C}$ and $12 \mathrm{D}$ ), cooperators organize into more compact clusters when no strategy exchanges are allowed $(p=0)$. This is a well known phenomena: structured populations allow cooperators to form clusters so that they interact mainly with each other, thus protecting themselves from exploration by defectors. This is important to understand how strategy exchanges influence the level of cooperation. A strategy exchange between a cooperator and a defector in the fringe of a cluster pushes the cooperator away from the other ones. At the same time, it introduces a defector inside the cluster or, at least, it contributes to more irregular cluster frontiers, which is also detrimental to cooperators (Nowak et al., 1994). This can be seen in Fig. 11 for the Prisoner's Dilemma game and in Figs. $12 \mathrm{~A}$ and $12 \mathrm{~B}$ for the Snowdrift game played under the GP rule. When strategy exchanges 

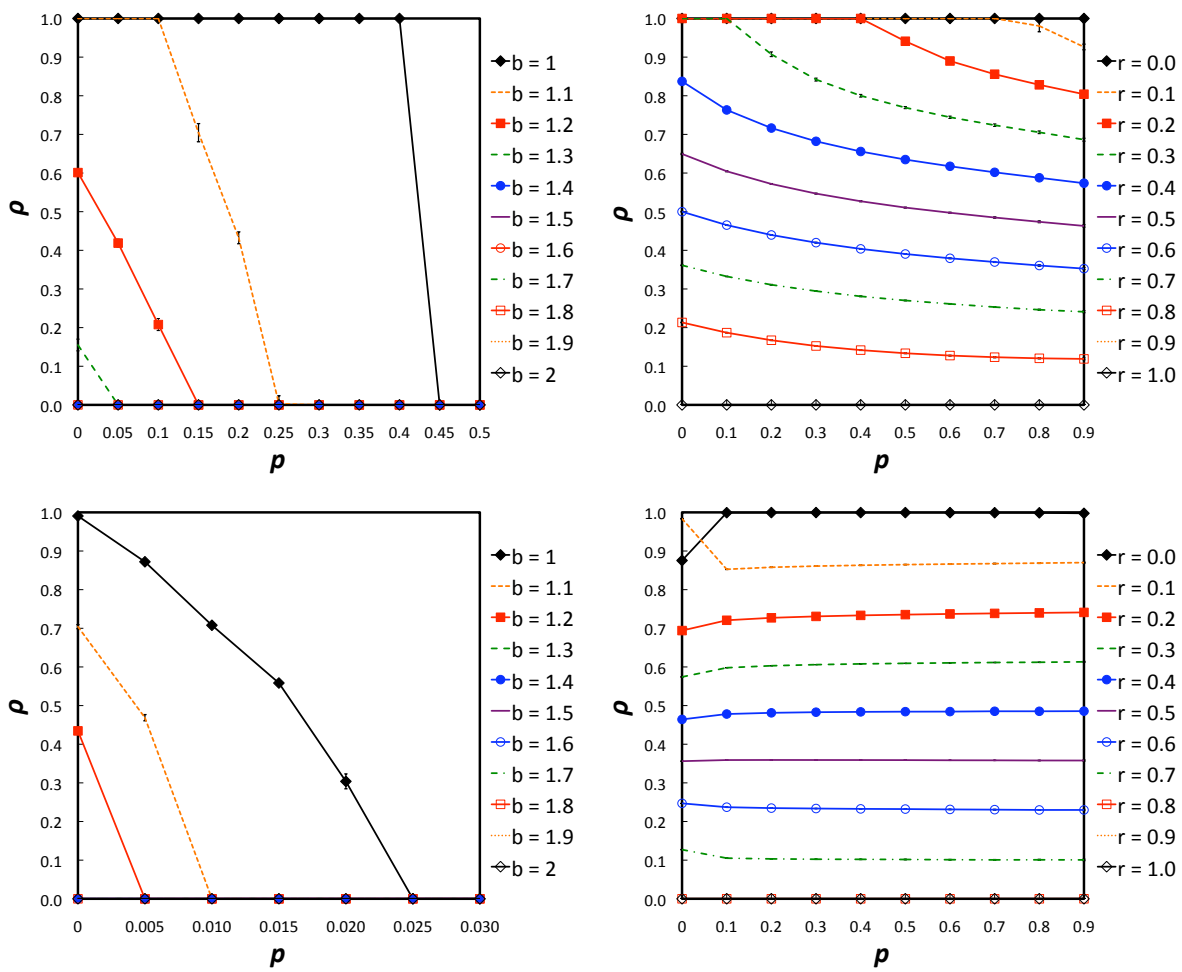

Figure 10: $\rho$ as a function of $p$ when the Prisoner's Dilemma (left column) and Snowdrift (right column) are played on regular networks $(\phi=0)$. Upper row: Moran rule (GP rule with $K=1$ ); Lower row: replicator rule. Results for $p=1$ in the Snowdrift game are not shown because in this regime the transition rule is never applied and, hence, payoffs do not influence the dynamics.

are introduced in the Prisoner's Dilemma game, cooperator clusters can not grow as they grow when no strategy exchanges occur. In the Snowdrift game played under the GP rule with $K=1$, cooperators scatter when strategy exchanges are introduced (Fig. 12 B). The situation is different when this game is played with the replicator rule (Figs. $12 \mathrm{C}$ and $12 \mathrm{D}$ ). In this case, agents do not organize into compact clusters even when there are no strat- 

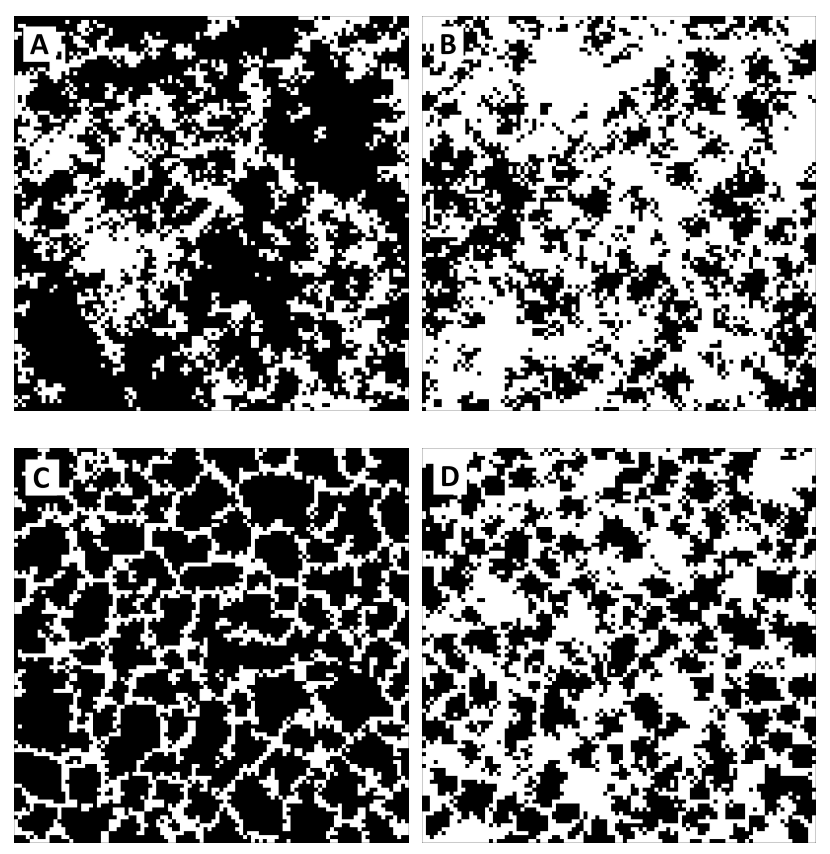

Figure 11: Typical steady state patterns when the Prisoner's Dilemma game is played on regular networks $(\phi=0)$. Upper row: Moran rule (GP rule with $K=1$ ) and $b=1.2$; Lower row: replicator rule and $b=1.1$. Left images: $p=0$; Right images: $p=0.05$ in panel $\mathrm{B}$ and $p=0.005$ in panel $\mathrm{D}$. Colors: black for cooperators and white for defectors.

egy exchanges (Fig. 12 C). This means that, when strategy exchanges are introduced (Fig. 12 D), there are no compact clusters to destroy and that is the reason why both the spatial patterns and $\rho$ are not affected.

This section shows that strategy exchanges prevent the growth of compact clusters of cooperators. Furthermore, the preceding section has shown that strategy exchanges are more frequent for larger synchrony rates, specially when noise is present in the strategy update process. This is a strong evidence that they are of major relevance concerning the beneficial effect of asynchronism in the presence of noise. 

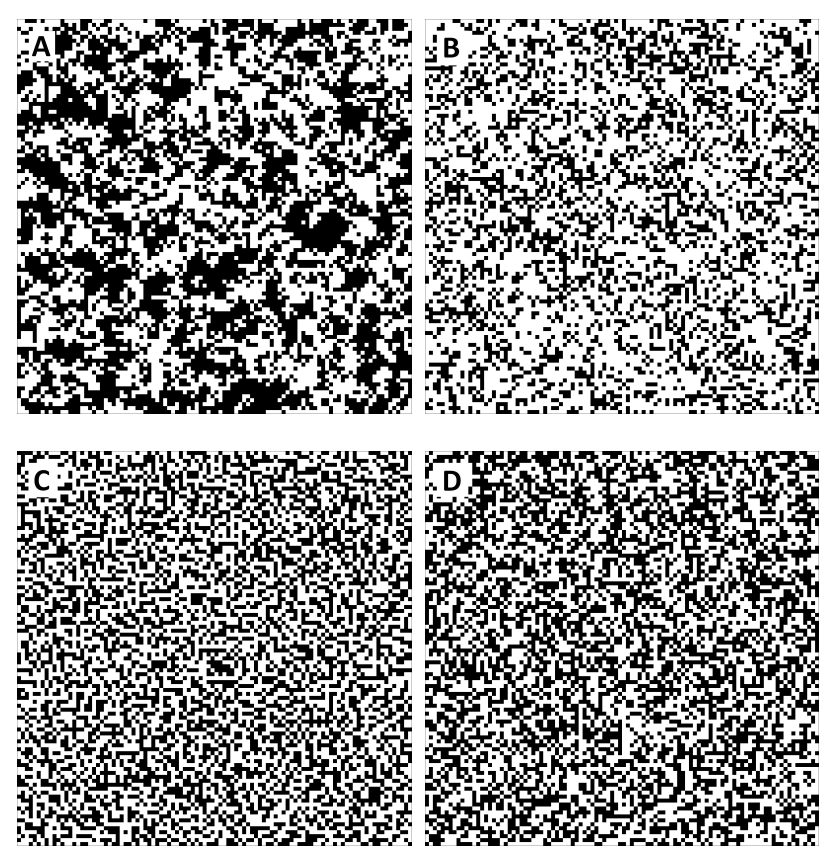

Figure 12: Typical steady state patterns when the Snowdrift game is played on regular networks $(\phi=0)$. Upper row: Moran rule (GP rule with $K=1$ ) and $r=0.6$; Lower row: replicator rule and $r=0.4$. The left images were taken for $p=0$ and the right ones for $p=0.9$. Colors: black for cooperators and white for defectors.

\section{Conclusion and Future Work}

This paper is a contribution to the understanding of the effects of the update dynamics on symmetric 2-player evolutionary games. It covers a broad number of conditions, allowing more general results to be achieved and provides a better picture of how and why they vary with the input conditions. The results show that, in general, asynchronism supports more cooperation. This is not valid only for very small noise values. Noise allows agents to make irrational choices, by imitating less successful neighbors. The replicator transition rule, which never allows agents to make such choices, 
is strongly insensitive to changes in the synchrony rate. Using a modified version of the Generalized Proportional transition rule, which prevents agents from making irrational choices, we have shown that this feature is a key property in the sensitivity to the update dynamics. This means that the influence of the update dynamics is strongly dependent on the noise level present in the update process and reinforces the conclusion concerning the beneficial effect of asynchronism on cooperation. It means also that, in order to build less sensitive artificial societies, special care should be taken in the design of strategy update processes and agents' perception skills, so that the imitation of less successful agents can be avoided or, at least, reduced. On the other hand, in systems where some noise is present, cooperation can be promoted through the maintenance of small synchrony rates by avoiding, for example, coupling mechanisms between agents or simply by refraining from forcing agents to update all at the same specific moments.

One of the side effects of the presence of noise in the update process is that direct strategy exchanges between agents become possible. The results show that, for larger noise values, there is a relationship between the occurrence of strategy exchanges, which decreases with the synchrony rate, and the way the update dynamics influences the emergence of cooperation. This is valid when games are played on small-world networks or on scale-free networks using average payoffs. In fact, we have found that there is a cause-effect relationship between strategy exchanges and the proportion of cooperators. An analysis of the spatial patterns formed by cooperator agents allows us to understand why this is so. Strategy exchanges are detrimental to the emergence of cooperation because they destroy compact clusters of agents when these exist, which is 
disadvantageous for cooperators. We have found also that there are situations where both the Prisoner's Dilemma and Snowdrift games are very sensitive to small changes in the synchrony rate, specially when we change from a synchronous dynamics to an almost synchronous dynamics. These situations occur mainly on scale-free networks using accumulated payoffs.

The work presented here focused mainly on the role of the transition rule on the influence of the update dynamics. However, the results show that the underlying network plays also an important role. The network models we used, while being among the most studied ones, do not allow us to identify the network properties that determine how the update dynamics influences the emergence of cooperation. This is because they are not sufficiently diversified concerning the properties by which networks are characterized. For example, the degree distribution of regular networks has a single peak while it follows a power law for scale-free networks. Furthermore, the regular networks used here have a large clustering coefficient while the Barabási and Albert scalefree networks present a very low one. This means that we do not know if the different results observed with regular and scale-free networks are due to the differences in degree distribution, clustering coefficient, both, or even other properties. In order to verify this, we need to study both regular networks with low or zero clustering coefficient and scale-free networks having larger clustering coefficients (Holme and Kim, 2002).

It has been shown that cooperation can be sustained when, besides strategies, the network of contacts also evolves. This has been shown analytically, considering that different types of agents seek new contacts and break existing ones at different rates (Pacheco et al., 2006a,b, 2008), as well as experi- 
mentally, when the influence that agents have on their neighbors may evolve (Szolnoki and Perc, 2008) or when the network topology may also evolve, both from scratch (Poncela et al., 2008) or from an initial network configuration (Zimmermann and Eguíluz, 2004; Szolnoki et al., 2008a). These works open new perspectives for the study of the influence of the update dynamics on the emergence of cooperation and, therefore, future developments of this work will consider coevolutionary games (Perc and Szolnoki, 2009). Other extensions will consider agents with memory (Lindgren and Nordahl, 1994; Kirchkamp, 2000) and multi-player games such as the Public-Goods game.

\section{Acknowledgements}

C. Grilo is supported by FCT/MCTES grant SFRH/BD/ 37650/2007.

\section{References}

Abramson, G., Kuperman, M., 2001. Social games in a social network. Phys. Rev. E 63, 030901.

Assenza, S., Gómez-Gardeñes, J., Latora, V., 2008. Enhancement of cooperation in highly clustered scale-free networks. Phys. Rev. E 78, 017101.

Axelrod, R., 1984. The Evolution of Cooperation. Penguin Books.

Barabási, A.-L., Albert, R., 1999. Emergence of scaling in random networks. Science 286, 509-512.

Bersini, H., Detours, V., 1994. Asynchrony induces stability in cellular automata based models. In: R. Brooks, P. M. (Ed.), Proc. Artificial Life IV Conf. MIT Press, pp. 382-387. 
Fatès, N., 2009. Asynchronism induces second order phase transitions in elementary cellular automata. J. Cellular Automata 4, 21-38.

Fatès, N., Morvan, M., 2005. An experimental study of robustness to asynchronism for elementary cellular automata. Complex Syst. 16, 1-27.

Gómez-Gardeñes, J., Campillo, M., Floría, L. M., Moreno, Y., 2007. Dynamical organization of cooperation in complex topologies. Phys. Rev. Lett. 98, 108103.

Gómez-Gardeñes, J., Poncela, J., Floría, L. M., Moreno, Y., 2008. Natural selection of cooperation and degree hierarchy in heterogeneous populations. J. Theor. Biol. 253, 296-301.

Grilo, C., Correia, L., 2009. The influence of the update dynamics on the evolution of cooperation. Int. J. Comput. Intell. Syst. 2, 104-114.

Hauert, C., 2002. Effects of space in 2x2 games. Int. J. Bifurcat. Chaos 12, $1531-1548$.

Hauert, C., Doebeli, M., 2004. Spatial structure often inhibits the evolution of cooperation in the snowdrift game. Nature 428, 643-646.

Helbing, D., 1992. Interrelations between stochastic equations for systems with pair interactions. Physica A 181, 29-52.

Hofbauer, J., Sigmund, K., 1998. Evolutionary Games and Population Dynamics. Cambridge University Press.

Holme, P., Kim, B. J., 2002. Growing scale-free networks with tunable clustering. Phys. Rev. E 65, 026107. 
Huberman, B., Glance, N., 1993. Evolutionary games and computer simulations. Proc. Natl. Acad. Sci. USA 90, 7716-7718.

Ingerson, T. E., Buvel, R. L., 1984. Structure in asynchronous cellular automata. Physica D 10, 59-68.

Kirchkamp, O., 2000. Spatial evolution of automata in the prisoner's dilemma. J. Econ. Behav. Org. 43, 239-262.

Lindgren, K., Nordahl, M. G., 1994. Evolutionary dynamics of spatial games. Physica D 75, 292-309.

Lumer, E. D., Nicolis, G., 1994. Synchronous versus asynchronous dynamics in spatially distributed systems. Physica D 71, 440-452.

Newth, D., Cornforth, D., 2009. Asynchronous spatial evolutionary games. BioSystems 95, 120-129.

Nowak, M., 2006. Evolutionary Dynamics. Harvard University Press.

Nowak, M., Bonhoeffer, S., May, R. M., 1994. More spatial games. Int. J. Bifurcat. Chaos 4, 33-56.

Nowak, M., May, R. M., 1992. Evolutionary games and spatial chaos. Nature $359,826-829$.

Ohtsuki, H., Nowak, M. A., Pacheco, J., 2007. Breaking the symmetry between interaction and replacement in evolutionary dynamics on graphs. Phys. Rev. Lett. 98, 108106. 
Pacheco, J. M., Traulsen, A., Novak, M. A., 2006a. Active linking in evolutionary games. J. Theor. Biol. 243, 437-443.

Pacheco, J. M., Traulsen, A., Novak, M. A., 2006b. Co-evolution of strategy and structure in complex networks with dynamical linking. Phys. Rev. Lett. 97, 258103.

Pacheco, J. M., Traulsen, A., Ohtsuki, H., Novak, M. A., 2008. Repeated games and direct reciprocity under active linking. J. Theor. Biol. 250, 723-731.

Perc, M., 2009. Evolution of cooperation on scale-free networks subject to error and attack. New J. Phys. 11, 033027.

Perc, M., Szolnoki, A., 2009. Coevolutionary games - a mini review. Biosystems 99, 109-125.

Poncela, J., Gómez-Gardeñes, J., Floría, L. M., Sánchez, A., Moreno, Y., 2008. Complex cooperative networks from evolutionary preferential attachment. PLoS ONE 3, e2449.

Roca, C. O., Cuesta, J. A., Sánchez, A., 2009a. Effect of spatial structure on the evolution of cooperation. Phys. Rev. E 80, 046106.

Roca, C. O., Cuesta, J. A., Sánchez, A., 2009b. Imperfect imitation can enhance cooperation. EPL 87, 48005.

Santos, F. C., Pacheco, J. M., 2005. Scale-free networks provide a unifying framework for the emergence of cooperation. Phys. Rev. Lett. 95, 098104. 
Santos, F. C., Pacheco, J. M., Lenaerts, T., 2006. Evolutionary dynamics of social dilemmas in structured heterogeneous populations. Proc. Natl. Acad. Sci. USA 103, 3490-3494.

Santos, F. C., Rodrigues, J. F., Pacheco, J. M., 2005. Graph topology plays a determinant role in the evolution of cooperation. Proc. R. Soc. Lond. B $273,51-55$.

Schlag, K., 1998. Why imitate, and if so, how? A bounded rational approach to multi-armed bandits. J. Econ. Theory 78, 130-156.

Schönfisch, B., de Roos, A., 1999. Synchronous and asynchronous updating in cellular automata. BioSystems 51, 123-143.

Szabó, G., Fáth, G., 2007. Evolutionary games on graphs. Phys. Rep. 446, $97-216$.

Szolnoki, A., Perc, M., 2008. Coevolution of teaching activity promotes cooperation. New J. Phys. 10, 043036.

Szolnoki, A., Perc, M., Danku, Z., 2008a. Making new connections towards cooperation in the prisoner's dilemma game. EPL 84, 50007.

Szolnoki, A., Perc, M., Danku, Z., 2008b. Towards effective payoffs in the prisoner's dilemma game on scale-free networks. Physica A 387, 2075-2082.

Szolnoki, A., Perc, M., Szabó, G., 2008c. Diversity of reproduction rate supports cooperation in the prisoner's dilemma game on complex networks. Eur. Phys. J. B 61, 505-509. 
Tomassini, M., Luthi, L., Giacobini, M., 2006. Hawks and doves on smallworld networks. Phys. Rev. E 73, 016132.

Tomassini, M., Luthi, L., Pestelacci, E., 2007. Social dilemmas and cooperation in complex networks. Int. J. Mod. Phys. C 18, 1173-1185.

Vukov, J., Szabó, G., Szolnoki, A., 2008. Evolutionary prisoner's dilemma game on Newman-Watts networks. Phys. Rev. E 77, 026109.

Watts, D., Strogatz, S. H., 1998. Collective dynamics of small-world networks. Nature 393, 440-442.

Weibull, J. W., 1997. Evolutionary Game Theory. MIT Press.

Winfree, A., 2001. The Geometry of Biological Time. Springer-Verlag.

Zimmermann, M. G., Eguíluz, V. M., 2004. Coevolution of dynamical states and interaction in dynamic networks. Phys. Rev. E 69, 065102(R). 